\title{
5 Jahre HeilberufeSCIENCE
}

\author{
Jörg Klewer
}

Online publiziert: 21. November 2013

(C) Springer-Verlag Wien 2013

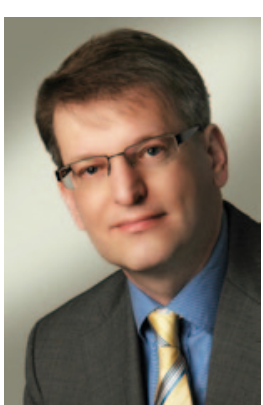

Jörg Klewer

Runde Geburtstage sind immer auch ein Anlass für Rückblenden - und wurden auch in der HeilberufeSCIENCEAusgabe 02/2013 von Reidl et al. wissenschaftlich betrachtet. Ferner bieten runde Geburtstage die Möglichkeit, Bilanz zu ziehen und in die Zukunft zu blicken. In der Bilanz stehen 5 Jahrgänge HeilberufeSCIENCE mit jeweils 4 Ausgaben/Jahr, dazu mehrere Kongress-Supplements anlässlich der Heilberufe-Kongresse in Hamburg und Berlin. Hinzu kommt die Listung in mehreren Datenbanken, u. a. auch CINAHL Complete. Dieser Erfolg eines neu etablierten wissenschaftlichen Journals mit „Blind-peer-review“-Verfahren gebührt primär den Autoren der veröffentlichten Beiträge und resultiert aus deren inhaltlicher Qualität.

Wie soll sich HeilberufeSCIENCE zukünftig weiterentwickeln? HeilberufeSCIENCE möchte die wissenschaftlichen Aktivitäten auf den Gebieten Pflegemanagement, Pflegepädagogik, Pflegewissenschaften, Gerontologie, Altenpflege und Gesundheitswissenschaften/Public Health gern weiter fördern und als Veröffentlichungsplattform für Studienergebnisse dienen. Insbesondere ist der Fokus auf den wissenschaftlichen Nachwuchs gerichtet, mit dem Angebot, über wissenschaftliche Kurzmitteilungen und „Online First“ interessante Forschungsergebnisse schnell zu veröffentlichen.

Deshalb finden Sie auch in dieser Ausgabe wieder 5 interessante Beiträge. Den Anfang macht eine englisch- sprachige Übersichtsarbeit von Quindemil et al., die den Forschungsstand zum Thema der familienzentrierten Pflege-Intensivstationen, v. a. hinsichtlich des Aspekts von Familienangehörigen mit Migrationshintergrund, referiert. Die nachfolgende Originalarbeit von Hirsch und Lindenberg analysiert die Beschwerden im Bereich des Bewegungsapparats bei Pflegekräften in einem Krankenhaus der Schwerpunktversorgung und unterstreicht den bestehenden Bedarf an gesundheitsfördernden Programmen für Pflegekräfte. Die Untersuchung von Schaal et al. setzt sich mit der Bewertung von Pflegeheimen mithilfe der Transparenzkriterien auseinander und weist darauf hin, dass die Optimierung von einrichtungsbezogenen Kriterien schneller zu einer besseren Beurteilung als die Optimierung von bewohnerbezogenen Kriterien führen kann.

Daran schließen sich 2 wissenschaftliche Kurzmitteilungen: Ebel et al. präsentieren den Aufbau einer Datenbank für Pflegekurse für pflegende Angehörige und zeigen auf, welche Vorteile eine solche Datenbank bietet. Die abschließende Studie von Hesse thematisiert die Anforderungen an das pflegerische Entlassungsmanagement eines Krankenhauses der Regelversorgung aus der Sicht nachsorgender Einrichtungen.

Abschließend möchte ich Sie wieder einladen, diese Ausgabe von HeilberufeSCIENCE kritisch und mit Interesse zu lesen. Nutzen Sie auch die Gelegenheit, sich anhand des zeitgleich erschienenen HeilberufeSCIENCE-Supplements mit den Abstracts der Poster und wissenschaftlichen Vorträge über das 4. HeilberufeSCIENCE-Symposium im Rahmen des 11. Gesundheitspflegekongresses zu informieren.

Zum Abschluss wünsche ich Ihnen eine schöne Weihnachtszeit und alles Gute für das Jahr 2014!

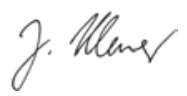

